This document must be cited according to its final version which is published in a conference proceeding as:

P. Dufour ${ }^{1}$, S. Bhartiya ${ }^{1}$, T.J. English ${ }^{1}$, E.P. Gaztke ${ }^{1}$, P.S. Dhurjati ${ }^{1}$, F.J. Doyle III $^{1}$,

"Faults detection of the continuous pulp digester",

Proceedings of the $4^{\text {th }}$ IFAC Workshop on on-line fault detection and super vision in the chemical process industries (CHEMFAS - 4), pp. 106-111,

Seoul, South Korea, june 7-8, 2001

All open archive documents of Pascal Dufour are available at: http://hal.archives-ouvertes.fr/DUFOUR-PASCAL-C-3926-2008

The professional web page ( $\mathrm{Fr} / \mathrm{En}$ ) of Pascal Dufour is: http://www.lagep.univ-lyon1.fr/signatures/dufour.pascal

1

University of Delaware,

Department of Chemical Engineering,

Newark, DE 19716, USA

http://www.udel.edu 


\title{
FAULT DETECTION IN A CONTINUOUS PULP DIGESTER
}

\author{
Pascal Dufour, Sharad Bhartiya, Thomas J. English, \\ Edward P. Gatzke, Prasad S. Dhurjati \\ and Francis J. Doyle III ${ }^{1}$
}

\author{
Department of Chemical Engineering, \\ University of Delaware, \\ Newark, DE 19716 \\ $U S A$
}

\begin{abstract}
:
Diagnostic strategies for Fault Detection and Isolation in a continuous pulp digester are presented. Several methodologies for Fault Detection and Isolation are compared for accuracy and real-time implementation potential. A Gross Error Detection methodology for biased and noisy measurements is initially examined. Next, a Gaussian Radial Basis Function neural network approach for detection of product quality related changes is also used. Changes in feedstock composition using an additional neural network approach is also developed. The efficiency and limitations of all methods are demonstrated using a first principles model.
\end{abstract}

Keywords: Diagnosis, Pulp and Paper Industry, Gross Error Detection, Neural Network.

\section{INTRODUCTION}

The pulp and paper industry forms a large and important sector of the Chemical Process Industry and is highly capital intensive. For the period 1996-1998, planned capital investments and new facilities to existing facilities were evaluated to exceed $\$ 10$ billion. Therefore, demand for higher safety and reliability has increased. Process diagnosis has gained considerable academic and industrial interest and numerous approaches have been proposed. Industrial control systems in this sector have become more complex, and advanced control systems like MPC require the presence of experts to diagnose the cause of performance degradation and to retune if needed.

The goal of our work is to develop and demonstrate computational modeling and control method- ologies that will facilitate integration of control and fault diagnosis methodologies for a continuous pulp digester. This paper is focused on methodologies and preliminary simulation results for Fault Detection and Isolation (FDI) as applied to this process.

The paper is organized as follows: a first principles digester model used in the different approaches is briefly described. Then, three diagnostic tools applied for the digester are discussed. First, an extension of a Gross Error Detection method is applied to the problem of biased and noisy temperature measurements. Then, a neural network approach dedicated to the qualitative detection of causes of changes in the final quality of the pulp, i.e., the blow line kappa number, is shown. Finally, another neural network approach to infer feedstock variations is presented.

1 Corresponding author: Fax: (302) 831 1048, Phone: (302) 831 0760, Email: fdoyle@udel.edu 


\section{CONTINUOUS PULP DIGESTER MODEL}

Continuous digesters (figure 1) are large vertical tubular reactors. White liquor (aqueous solution of effective alkali and hydrosulfide) strips the presteamed porous wood chips of lignin, freeing the wood fibers. The main reaction takes place in the upper section of the digester referred to as the cook zone. In this section, both the chips and liquor flow cocurrently. At the end of the cook zone, spent liquor is extracted for chemical recovery. The chips, however, continue their downward flow in the modified continuous cooking (MCC) zone and extended modified continuous cooking (EMCC) zone where they encounter weak liquor flowing countercurrently. The wood pulp is finally extracted at the outlet of the EMCC. The main control issue is concerned with the quality of the pulp, i.e., the kappa number.

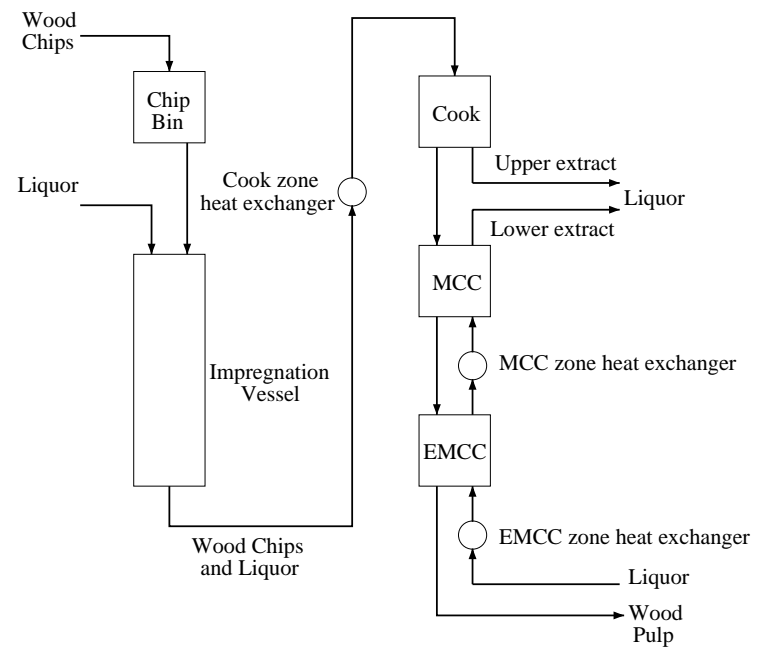

Fig. 1. Schematic of a chemical pulp digester.

For modeling purposes, the digester is approximated as a series of 150 continuous stirred tank reactors (CSTR). The first principles model consists of mass balances for the non-porous solid and the free liquor components as well as balances between the 2 phases within each reactor. For modeling assumptions and a description of the conservation laws, the reader is referred to (Wisnewski et al., 1997). Based on this model, a code using Matlab has been developed (Doyle et al., 1999) and is adopted to simulate the process and the model in the various approaches developed in this paper.

\section{GROSS ERROR DETECTION}

In modern plants, a large number of measurements are available. Their accuracy has an impact on process monitoring, model identification and control. This section is concerned with the problem of noise and bias in temperature measurements. A Gross Error Detection method is investigated: this method is suited to identify and eliminate measurements containing systematic biases. It was initially developed for linear steady state models (Narasimhan et al., 1987). The idea is to evaluate the unknown bias vector $b$ and true measurement vector $y$ by solving:

$$
\min _{y, b}\left(y_{p}-b-y\right)^{T} Q^{-1}\left(y_{p}-b-y\right)
$$

subject the constraints:

$$
A y=C
$$

where $y_{p}$ is the vector of the biaised and noisy measurements, $Q$ is the known noise covariance, and $A y=C$ represents the steady state constraints.

This framework has been extended in various approaches for a nonlinear steady state model (Renganathan et al., 1999): simulation results in chemical engineering showed that as the nonlinearity of the system in increased, the proposed test lead to better results rather than tests which rely on linearizing the model.

In this section, we propose an extension of the initial approach to nonlinear dynamic system. This requires the solution of:

$$
\min _{y, b}\left(y_{p}-b-y\right)^{T} Q^{-1}\left(y_{p}-b-y\right)
$$

subject to the model resolution:

$$
\left\{\begin{array}{l}
\dot{x}=f(x, u) \\
y=g(x)
\end{array}\right.
$$

In the current continuous digester, four temperature measurements are assumed to be subjected to noise and bias. These sensors are located at the upper and lower extracts and at the ends of the EMCC zone. The results depicted in figure 2 show that all the real unknown measures can be estimated despite the bias and noise introduced at different times.

However, since an on-line optimization problem that includes the model resolution has to be solved, computational time can become an important issue during implementation, as depicted in figure 3 after 3 hours of simulation (the sample time is 10 minutes). This can seriously limit implementation of this method, especially when a more complex model is employed. 

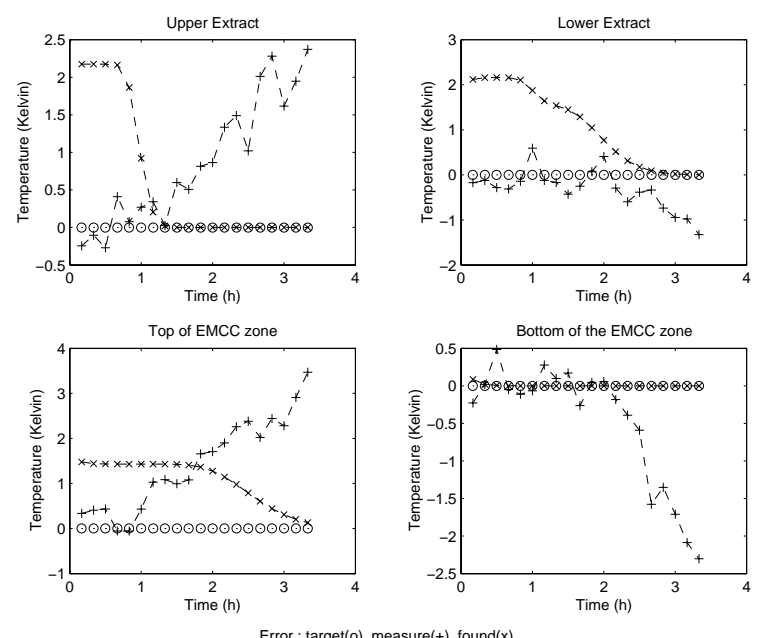

Fig. 2. Reconstruction of 4 temperature measurements.

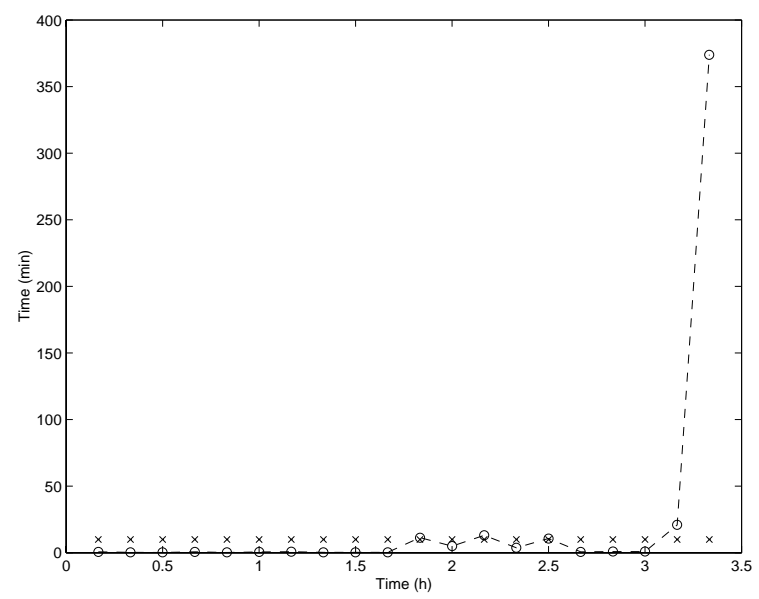

Fig. 3. Computational $(o)$ and sample times $(\times)$.

\section{PULP QUALITY VARIATIONS DETECTION}

In this paper, two different uses of a neural network are investigated. The first one is concerned with the variations of the kappa number, i.e., the pulp quality index at the extraction of the digester vessel. The problem is that different changes occuring in physical properties at various locations accross the digester can similarly affect the final kappa number at the outlet of the digester. Four of them are concerned with:

- The lignin increase in the feed chips that causes the kappa number to increase because more lignin has to be removed during the chemical reaction.

- Moisture content increase in the feed chips dilutes the liquor, thereby reducing the rate of reaction and also the amount of lignin removed and hence the kappa number increases.

- Liquor introduced in the impregnation vessel is coming from a recovery loop. The decrease of the density of one of its two species, the hydrosulfide (HS), makes the reaction rate decrease and then the kappa number increases.

- The reduction of the temperature in the cook zone decreases the reaction rate, reducing the amount of lignin removed and a consequence rise in the kappa number.

To normalize the fault signatures, the slope of each ramp for the implementing variable is adjusted so that a kappa number increase of 2 after 24 hours occurs at precisely the same hour for each fault. Therefore, the sole examination of the kappa number does not indicate which of these variables has changed.

Neural networks have been used for over 15 years for modeling and control as well as for fault detection (Belsito et al., 1998) (Yu et al., 1999). Another approach combining a neural network and a fuzzy system improves the diagnosis time and performance for a qualitative feedstock variations detection in a chemical plant (Ruiz et al., 2001).

The approach used here is a three nodes gaussian radial basis neural network, where each node corresponds to a fault. Each node is trained to respond with a 0 if there is no fault and a 1 if this particular fault occurs: the neural network is constructed here in a qualitative manner. The cause of this kappa number variation is inferred from 16 simulated plant measurements:

01. Cook zone heat exchanger temperature

02. MCC zone CSTR \# 16 temperature

03. EMCC zone heat exchanger temperature

04. Effective alkali Cook out

05. Effective alkali MCC out

06. Effective alkali EMCC out

07. Hydrosulfide Cook in

08. Hydrosulfide Cook out

09. Dissolved solids Cook out

10. Dissolved solids EMCC in

11. Kappa Cook in

12. Kappa Cook out

13. Kappa EMCC out

14. EMCC liquor volumetric rate

15. Dissolved lignin MCC in

16. Dissolved lignin EMCC out

Moreover, because neural network construction requires a training data set, different rates of change for the implementaion variable were used to create the training data from the plant simulator.

Several cases studies with the final 3 node neural network are now presented where each simulation is run and a fault begins at 10 hours in the corresponding implementation variable. Using the neural network in the trained situations depicted 
in figure 4a for the HS density variation and in figure $4 \mathrm{~b}$ for the moisture content variation leads to promising results: in spite of some errors in the 2 other nodes when the fault starts, the third node is able to recognize the origin of the fault.
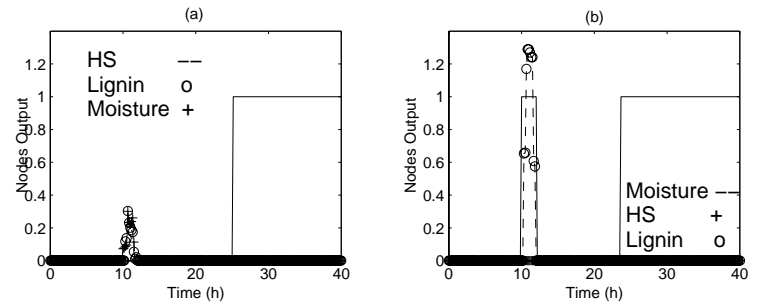

Fig. 4. Neural Network Responses to trained fault: (a) HS, (b) Moisture

The generalization of the 3 node neural network can also be seen for an untrained situation: in figure $5 \mathrm{a}$, a reduction of the cook temperature leading to an increase of the kappa number has been introduced. The neural network outputs behave well since one of the three faults is persistently recognized. However, the natural limitation of the neural network to extrapolate to untrained regions can be noticed in figure $5 \mathrm{~b}$ : while the training set has been built with 23,24 and 25 hours ramps, the network is unable to detect the 21 hour ramp in HS density.
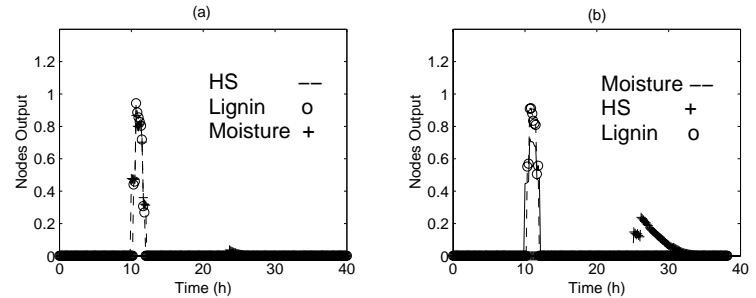

Fig. 5. Neural Network Responses to untrained faults: (a) Cook Temperature, (b) HS

A more general evaluation of this neural network shows that $74 \%$ of the fault cases were correctly diagnosed. The influence of the structure of the neural network (Henrique et al., 2000) is also emphasized here: a single node neural network has also been constructed (giving 4 different values 1 , 2 and 3 for the three different trained faults and 0 if none). It was able to diagnose correctly only $54 \%$ of the faults.

It can be concluded that gaussian radial basis neural network is capable of detecting such variations.

\section{FEEDSTOCK VARIATIONS DETECTION}

This section addresses detection of unmeasured variations of feedstock properties in the inlet of the digester (figure 6) that affect the final kappa number, even in a MPC structure (figure 7)
(Wisnewski et al., 1998.). Precisely, theses changes consist of:

- Moisture content that is measured once a day,

- unmeasured densities of the five wood species fed in the chip bin: high reactivity lignin, low reactivity lignin, carbohydrate, galactoglucomman and araboxylan, and,

- unmeasured densities of the two liquor species fed in the impregnation vessel, i.e., the effective alkali(EA) and the hydrosulfide(HS).

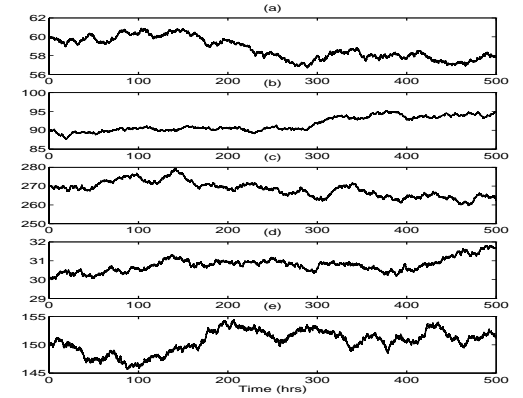

Fig. 6. Stochastic wood densities compositions $\left(\mathrm{kg} / \mathrm{m}^{3}\right)$ : (a) high and (b) low reactivity lignin, (c) carbohydrate, (d) araboxylan, (e) galactoglucomman
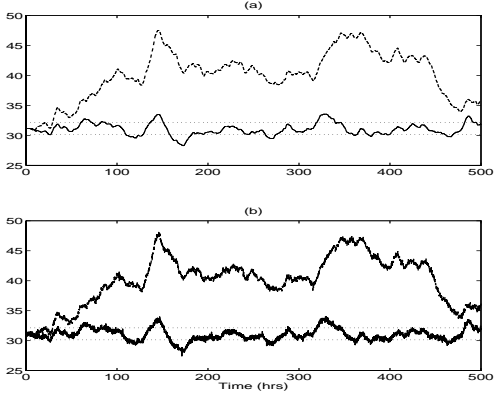

Fig. 7. Kappa number in open loop (dashed) and with MPC (solid): (a) no noise, (b) with noise

The method developed here is based on a neural network. The idea lies in inferring the value of these eight unknowns using real available measurements present in the digester. Moreover, the use of an accurate model is crucial in this case, since these eight feedstock qualities descriptors are not available and therefore their effects can not be quantified.

A sensitivity analysis shows that, among all the sensors actually available, the EA and HS measurements of the liquor at the upper extract of the digester are the most useful to detect the causes of the changes in the kappa number. They will both be used to infer the eight unknown values in the neural network strategy.

The first issue concerned with the training of the neural network is the construction of the training set: i.e, how to choose the variation set defined by the values that can take the eight simulated 
unknowns? And which of them can be inferred by this method?

An initial neural network was trained using simple cases to construct the variations set: each of the eight unknowns can take eight different values about its nominal value (100\%) but all the changes that occur have the same step magnitude. This involves the computation of $2^{8} \times 8=2048$ simulations that last 12 hours, i.e., sufficiently long such that the EA and HS measurements at the upper extract are affected by these changes. Using this neural network in trained situations allows one to conclude that the moisture content (figure 8a), the carbohydrate density (figure 8b), the araboxylan density and the HS density can be inferred.

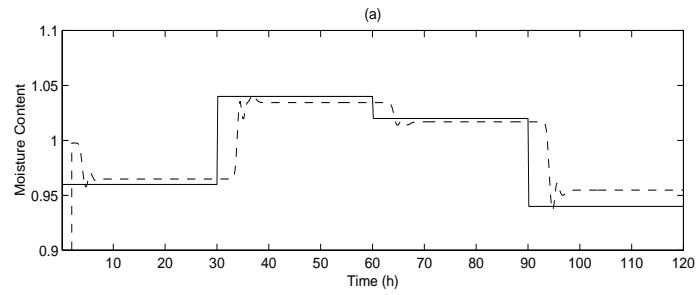

(b)

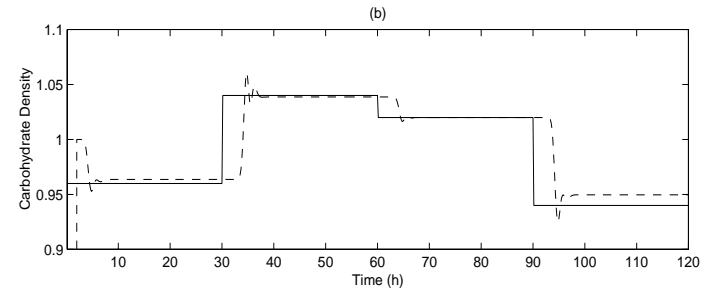

Fig. 8. Moisture content (a) and Carbohydrate density (b): normalized variations introduced (solid) and neural network response (dashed)

Using this neural network in untrained regions (here with uncorralated variations for each of the eight variables) shows its limitations: the results are very poor (figure 9). This underlines the problem of constructing the variations set.

Since the measurements used to feed the neural network are sensitive to three candidate manipulated variables (MV) of the control structure, these MVs have to be introduced in the variation set: the chips flow rate at the inlet of the impregnation vessel, the liquor flow rate at the upper extract and the cook temperature. Moreover, as only four of the eight unknowns seem to be reconstructable by the neural network and in order to reduce the amount of data needed to create the training set, only the four most detectable unknowns are used.

The idea is now to build a variation set for each of the unknowns as follows: it is assumed that only the three MV and also the relevant unknowns can vary among nine different values. This leads to $9^{4}=6561$ different simulations where only step changes are introduced to produce the variations.
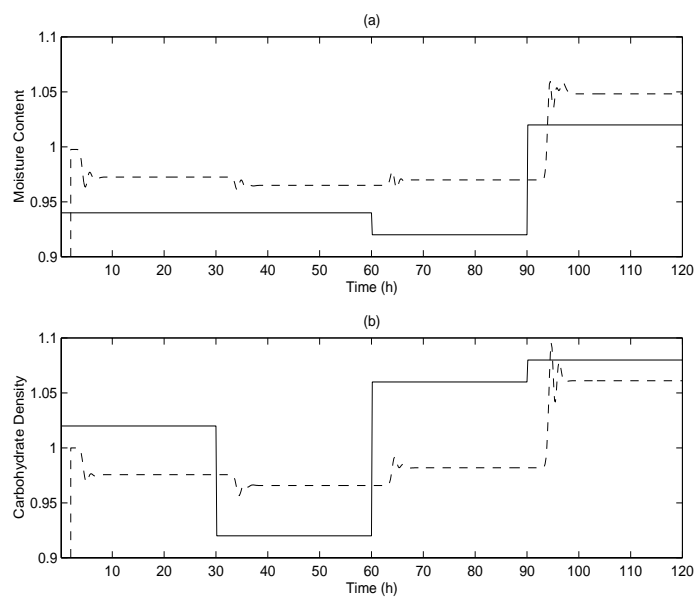

Fig. 9. Moisture content (a) and Carbohydrate density (b): normalized variations introduced (solid) and neural network response (dashed)

To avoid computational issues encoutered in the neural network creation, only 2000 of them are used randomly to create the training set.

Using this neural network in trained situations leads to good results. The extension to untrained behaviors (figure 10) where the changes have a first order shape leads to interesting results (figure 11): in spite of transient errors, the tracking of the variations introduced during the simulation is effective. More interesting is the use of the neural network to another untrained situations: a suite of changes for each of the seven others unknowns are introduced (starting from the moisture content figure $12 \mathrm{a}$ to the EA density figure $12 \mathrm{~b}$ ). It can be seen that the neural network can naturally reject these disturbances (figure 13), except for the moisture content (the first change) and the carbohydrate (the fourth change). Since both of these variables can be inferred using other neural network, theses results could be combined to correct these errors. Otherwise, after transient errors, the normal value is nearly reached again.
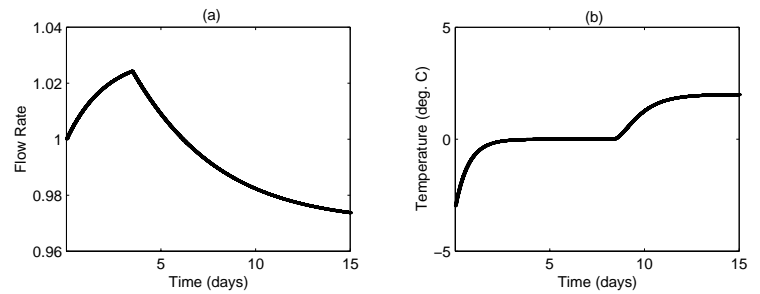

Fig. 10. Neural Network Responses to untrained faults: (a) Cook Temperature, (b) HS

\section{CONCLUSION}

Three different approaches for the diagnosis of a pulp digester have been evaluated based on a first principles model. For the Gross Error Detection, even if good results have been obtained, on-line 


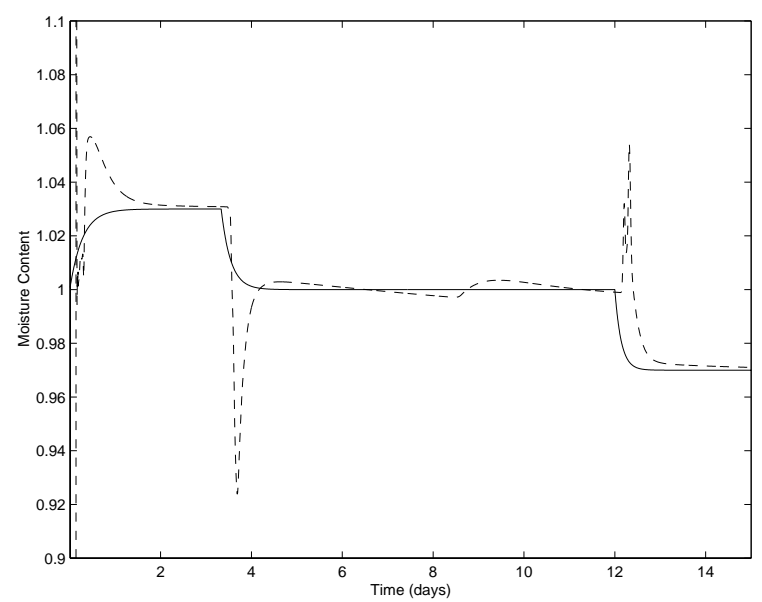

Fig. 11. Moisture: normalized variations introduced (solid) and neural network response (dashed)
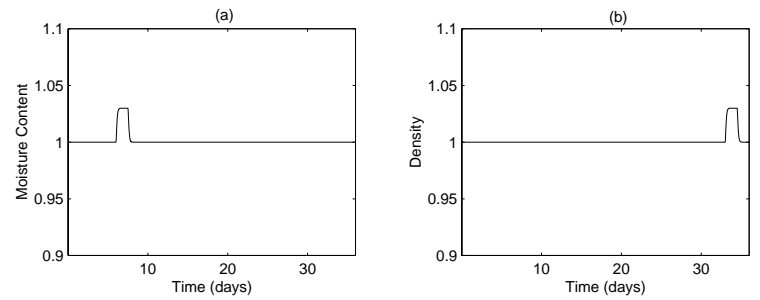

Fig. 12. First (a) and last (b) changes introduced in the suite

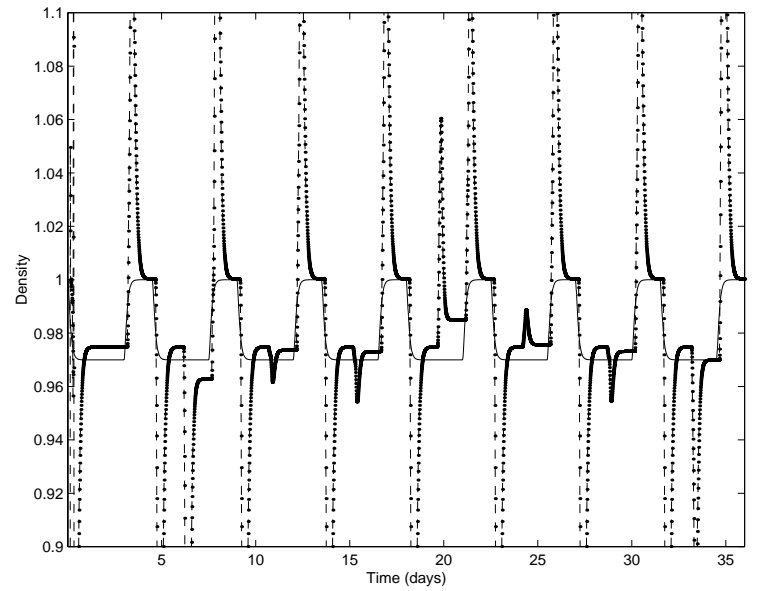

Fig. 13. HS: normalized variations introduced (solid) and neural network response (dashed)

computational time can restrict the use of this method. This limitation is not present with the neural networks since the necessary training is performed off-line. A first neural network method has shown good potential to detect changes in properties at the inlet of the digester. A second neural network approach leads to good results to infer changes in the moisture content, the density of two wood species and one density of the white liquor, even with untrained situations. A potential problem in the choice of the variation set leading to the construction of the training set has also been pointed out. Future developments are related to a Moving Horizon Estimation strategy (Gatzke et al., 2001) for the feedstock variation problem and with the on-site evaluation of these methods.

\section{ACKNOWLEDGMENTS}

The authors acknowledge funding from the Departement Of Energy (Grant DE-FC07-00ID13882).

\section{REFERENCES}

Belsito S., Lombardi P., Andreussi P.and Banerjee S. (1998). Leak Detection in Liquified Gas Pipelines by Artificial Neural Network. AIChE J. Vol. 44(12), pp.2675-2688

Doyle III F.J. and Kayihan F. (1999). Reaction Profile Control of the Continuous Pulp Digester. Chemical Eng. Science Vol. 54. pp. 2679-2688

Gatze E.P and Doyle III F.J. (2001). Use of Multiples Models and Qualitative Constraints for on-line Moving Horizon Disturbance Estimation and Fault Diagnosis. J. Process Control, in press 2001

Henrique H.M., Lima E.L. and Seborg D.E (2000). Model Structure Determination in Neural Network Models. Chem. Eng. Sci. Vol. 55, pp. 5457-5469

Narasimhan S. and Mah R.S.H. (1987). Generalized Likelihood Ratio Method for Gross Error Identification. AIChE J. Vol. 33, 1514

Renganathan T. and Narasimhan S., (1999). A Strategy for Detection of Gross Errors in Nonlinear Processes. Ind. Eng. Chem. Res. Vol. 38. pp. 2391-2399

Ruiz D., Nougues J.M and Puigjaner L. (2001). Fault Diagnosis Support System for Complex Chemical Plants. Comp. 6 Chem. Eng. Vol. 25, pp 151-160

Wisnewski P.A., Doyle III F.J. and Kayihan F. (1997). Fundamental Continuous Pulp Digester Model for Simulation and control. AIChE J. Vol. 43, pp. 3175-3192

Wisnewski P.A. and Doyle III F.J. (1998). Control Structure and Model Predictive Control of the Weyerhauser Digester Problem. J. Proc. Control Vol. 8, pp. 487-495

Yu. D.L., Gomm J.B. and Williams D. (1999). Sensor Fault Diagnostic in a Chemical Process via RBF Neural Networks. Cont. Eng. Pract. Vol. 7, pp. 49-55 